\title{
Optimal Wage Distribution in Hierarchies
}

\author{
Francesc Dilmé i Soto \\ Departamnent de Teoria Economica \\ Universitat de Barcelona (UB), Catalonia, Spain \\ francesc.dilme@gmail.com
}

\begin{abstract}
Most of the large firms organization schemes consist in hierarchical structures of tiers with different wage levels. Traditionally the existence of this kind of organizations has been associated to the separation of productive and managerial or supervision tasks and differences in the skills of the workers. Nevertheless, in the present there are in firms with similarly skilled workers where the hierarchical structure could be related to an incentives scheme to make the agents work. The model we present tries to inquire how the firm owner should decide the optimal wage distribution in order to maximize the profits obtained by the firm.
\end{abstract}

Keywords: Optimal wages, hierarchies, firm structure, incentives scheme, moral hazard.

\section{INTRODUCTION}

The classical principles of Economics, since the D. Ricardo (1817) time, say that the wage earned by a worker should approximate, in a competitive free market economy, to his or her marginal production. This statement is valid when it is easy to calculate the marginal product of a worker and there are few management tasks as, for example, in small size firms. 
Nowadays a great portion of the society is employed in large firms, that many times have an international scope, with thousands of employees around the world. In fact, many economic historians, as Chandler (1962), have documented that an important part of the economic development in the last centuries has been driven by large scale organizations, some of them with hundreds of thousands of employees. In these large firms is difficult to calculate the marginal production of every individual employee and the effort he or she supplies. Moreover, the wage is usually contracted before the performance of the productive process, and usually all employees with the same rank earn the same wage regardless the effort supplied.

The production of these large firms depends on the effort supplied by their employees, that many times is a hidden action, not easily observable by the firm owner. This fact provides incetives to the employees to shirk. Dickens, Katz, Lang and Summers (1989) present extensive evidence of the importance of worker shrinking in the firms of United States. By his hand, Akerlof and Yellen (1990) show the correlation between worker's effort and forces like anger, jealousy and gratitude. Moreover, in the large firms is difficult to make contracts linking the wage to the overall production of the firm. Nevertheless, this possibility does not solve the problem, because when the number of workers is large the performance of the whole firm is not linked to the individual effort.

In most of the moral hazard literature it is assumed that the cost of supervising the effort supplied by the employees is quite large. This circumstance generates a situation with asymmetric information between the firm owner and the employee, where only the employee knows the effort he or she supplies, the «hidden action». Then the effort, as a non measurable variable, usually can not be included in the contracts between the principal and the agent. The elaboration of incentive mechanisms to increase the effort supplied of the agents to be competitive is an important issue for the large firms.

A first possibility to provide motivation to the workers to supply effort is through punishment schemes that try to detect shirking and penalize this behavior, commonly named «stick-schemes». These schemes consist with stochastic inspections to the workers and penalizations if they are found shirking, that usually consist in firing them. There are models like Shapiro and Stiglitz (1984) that present where the firms have limited monitoring abilities and force the workers to exert effort through a stochastic process that allows the detection of shirking at a certain probability rate. Nevertheless, it is usually difficult to have irrefutable proofs that the worker has been shirking continuously, and without these proofs the legal system does not allow to fire a worker. 
Qian (1994), following Calvo and Wellisz (1978,1979), develops a model of optimal hierarchical organizations. These models treat the hierarchies as supervisory schemes where in the bottom tier of the firm there are the productive workers, that produce the good that is sold by the firm. The rest of tiers is a system where every agent supervises the agents in the immediately lower tier. The optimal structure tries to prevent the «loss of control», that is, the lack of supervision of the lower tiers. This vision is far of being realistic in present firms, specially those that belong to the services sector, where the tasks of upper tiers of the hierarchy are varied and often not specially correlated in monitoring the effort of the lower tiers agents.

The other possibility of make the agents supply effort is through positive incentives, commonly named «carrot schemes». The optimal contracts theory provides different contracts in situations where the agents supply effort that is not directly observable by the principal. Then, the principal pays to the agent the information rent, related with the fact that the agent knows information unknown by the principal (the effort he or she supplies). This model is not always applicable, because of its the assumptions not always hold. It is usually hard to know about the probabilities of the different states of the nature, some kinds of the contracts are not legally allowed,...

Holmstrom (1982) formalizes the model of Alchian and Demsetz (1972) of optimal contracts in cases of moral hazard in teams, treating the firm as essentially a team where only the aggregated output is observable. The principal objective of this kind of contracts is to avoid the free rider worker, than receives a wage without doing effort. By his part, Tirole (1986) develop the collusion-proof contracts, in order to avoid collusion among agents or among the supervisors and the workers. These models are interesting in the contract theory, but they do not explain the hierarchical structure of the firms.

Lazear and Rosen (1981) and Green and Stokey (1983) develop the optimal design of tournaments, modelling the incentives to work as lotteries with prizes. In this kind of lotteries, the probability of winning the prize depends stochastically on the effort performed by the contestants. In this model, the contestants decide the effort they supply and after the nature alters stochastically the result of their «investment». Finally a prize is given to the player that has the high observable investment. Then, the probability of winning the lottery depends on the effort individually supplied, but in a stochastic way. This game of tournament can only be solved analytically when only two players are considered, but it is not consistent with most of the upgrading procedures in large firms. Moreover, the Lazear-Rosen model does not explain the hierarchical wage structure of the firms, and wage contracts, that many times are uncorrelated to the production, are usually non treatable as prizes to the production. 
In the large firms, that usually have highly hierarchical structures, the agents usually have a stronger incentive to work: to be upgraded. In fact, many large firms have a structure based in different tiers with increasing wages. In this structure, when a new worker is hired, he or she begins in the lowest tier of the firm, and supplying effort he or she is upgraded with some probability. The incentive of higher future salaries provides to the workers high incentives to effort, specially when competition to be upgraded among workers plays an important role. Then, upgrading mechanisms where the probability of success depends on the own supplied effort can incentive the agents to work. The incentive of increase the utility usually comes from the possibility of being upgraded, that is, the incentive of achieve a new wage level.

Many times the necessity of a hierarchical structure in the large firms does not only arise from the necessity of management tasks, but also from the necessity to provide incentives to the agents to supply higher levels of effort. This incentive-based wage structure usually implies that agents will be payed under their marginal production in the lower tiers and agents payed above their marginal production in the higher tiers. In fact, there are many firms where the high wage increases from one tier to the next one make hardly conceivable that the productivity of an agent that is upgraded increases in accordance with them. Moreover, it is often possible to find highly skilled workers in the job market or in lower tiers capable of supplying similar productivity than the agents of the high tiers for lower wages, but firms do not low the wages of the higher tiers in order to maintain the incentives of their agents.

We now propose a model based in this observable behavior of the firms and the agents. We assume that when there is a vacancy in a certain tier on the salary ladder there is a lottery among the agents in the lower tier to occupy this vacancy. The probability that each agent has to win this lottery depends on the effort supplied by him or her.

This paper is organized in 5 sections. After this introduction, in the second section the general the model is established, analyzing the production, the probability of being upgraded and the moral hazard. The third section presents a particular case, where the agents can only choose among two efforts. In the fourth section the symmetric Nash equilibrium is solved. In the fifth section there are many comparisons of the results previously obtained, specially comparing the two particular cases and also the different scopes of the problem. In this section we also will treat inequality issues. Finally, we will present the conclusions that can be obtained from the results presented. 


\section{THE MODEL}

\subsection{Production}

Let's establish first the production side of the firm. We assume that the firm, through the effort of the agents, produces an homogeneous product, that is sold at a nominal price $p>0$ taken as given by the firm. We also assume that there are no other production costs than the wages payed to the agents. This assumption is equivalent to suppose that the costs other than the wages are proportional to the production, and the net revenue of every unit of good is its price. The firm owner, then, have as nominal revenue the overall production of the firm multiplied by the price of this product, and the wages payed to the workers as costs.

We define a firm as a structure $Q \in N$ of jobs organized in $N \leq Q$ subsets or tiers, each one with a large number of jobs given by $Q_{n}$, and with an associated wage, $w_{n}$, with $n=1, \ldots, N$. We assume that the wages are arranged in rising order, $w_{1} \leq \ldots \leq w_{N}$. At every instant of time $Q$ individuals, called agents, belong to the firm, and occupy one and only one job. Every job belongs to one tier, and then $\sum_{n=1}^{N} Q_{n}=Q$. We will assume that the wages that the firm owner can establish have a lower limit, given by a minimum wage $w_{b}$. This minimum wage can be established by the government, for example through minimum wage law, or by the market, for example as an opportunity cost of the agents.

Every agent can supply an effort given by ${ }^{1} \lambda^{i} \in R^{+}$. We assume that the production depends linearly on the effort through a productivity that depends on the tier where the agent works. The real productivity per unity of effort worked by an agent of the tier $n$ will be noted by $A_{n}$, and then the production of an agent that belongs to the tier $n$ and supplies an effort $\lambda^{i}$ is $A_{n} \cdot \lambda^{i}$. The nominal productivity is given by the product of the real productivity and the price of the good. The overall nominal production $Y^{\text {nom }}$ will be given by the following function

$$
Y^{\text {nom }}=\sum_{n=1}^{N} p \cdot A_{n} \cdot \bar{\lambda}_{n} \cdot Q_{n}
$$

${ }^{1}$ From now we note $R^{+}$as the non negative reals, including the 0 . For the moment we assume en effort without upper bound, that can seen non realistic, but as we will see it will be naturally bounded. 
where $\bar{\lambda}_{n}$ is the average effort supplied by the agents in the tier $n$. Because the firm owner can not observe the effort supplied by the agents, he or she pays the same wage to all the agents belonging to the same tier, given by $w_{n}$, regardless the effort they supply. Then, the nominal production costs $C^{\text {nom }}$ are given by

$$
C^{\text {nom }}=\sum_{n=1}^{N} w_{n} \cdot Q_{n}
$$

The nominal profits of the firm are given, as usual, by the nominal production minus the nominal costs. Then, the profits function $P^{n o m}$ is given by the following formula:

$$
P^{\text {nom }}=Y^{\text {nom }}-C^{n o m}=\sum_{n=1}^{N}\left(p \cdot A_{n} \cdot \bar{\lambda}_{n}-w_{n}\right) \cdot Q_{n}
$$

The firm owner faces the problem of maximize this nominal profits function. In general he or she will decide the wages of all the tiers, with the constraint of being higher or equal than the minimum wage $w_{b}$. We will consider additionally the case where the firm owner can also choose the firm organization, that is, the number of agents in every tier $\left\{Q_{n}\right\}$, and maximize the profits optimizing also these values. Then, in general, the firm owner can not decide the average effort of every tier, because every agent decides the effort he or she supplies individually and it can not be observed, but can influence the decisions through changes in the incentives to win the lotteries, changing the wages and the firm organization.

All the nominal variables we have just presented can be translated to the real analogous variables just dividing them by the general price level of the economy. We will consider that the production of the firm represents an small proportion of the overall production of this good, and that this good represents only a small portion of the consumer basket. Then, changes in the price of the product without changes in the nominal wages will not affect the real wage of the workers of the firm. Nevertheless, when later in the paper we will consider the whole economy and the aggregated production, the price of the aggregated production will represent the general price level, and then the real variables will be strongly dependent of the price. 


\subsection{Probability of being upgraded}

We assume that the agents that belong to the firm can leave it, process that will be called «death». An agent then can «die» due the corporeal death, but also because the agent reaches the retirement or the agent decides to change the firm where he or she works. We make the simplifying assumption that the death rate is the same by all the agents that work into the firm, regardless their age or the tier they belong. Then, the process of dying can be treated as a stochastic exponential process of constant rate, that will be noted by $d$. At every moment of time, the probability of every agent of surviving a time $\tau$ from this moment is $e^{-d \cdot \tau}$, and in particular, when $\tau=\Delta t$ is small, the probability of dying can be approximated by $d \cdot \Delta t$. The life expectancy of every agent in the firm is $1 / d$.

When an agent that belongs to the firm dies he or she leaves a vacancy in his or her tier. We assume that this vacancy can only be occupied by an agent of the immediately lower tier through a mechanism of selection, that will be treated later in this paper. We also assume that the agents can not be degraded or fired ${ }^{2}$. Then, in every tier the vacancies can only be produced by the upgrading of an agent from this tier to the immediately upper tier or by the death of an agent of this tier. It implies that, when an agent that belongs to certain tier dies, there is a cascade effect, producing an upgrading chain of all the tiers lower than this one. When there is a vacancy in the lowest tier, the tier 1 , one worker is automatically hired by the firm.

The assumption of automatic hiring of an agent can be understood as the existence of a minimum wage established by the government and an unemployed pool of agents that are eager to work at the wage $w_{1} \geq w_{b}$. In this case, we can assume that their reserve utility is sufficiently low to accept immediately a job in the firm. Another possible assumption is to assume a reserve utility established by the opportunity that the agents in the labor market have. Then, we can assume that there is no minimum wage and the only restriction for the wages is that they should be non negative. The results in this case are similar to the establishment of the minimum wage by the government, and we will obviate this case.

2 Examples of the impossibility of fire workers are the functionaries, that can not be fired except in extreme cases, and labor laws that do not allow to fire workers except when there are irrefutable proofs of their shirking, that are difficult to obtain. 
Let's now inquire about the probability of being upgraded at a certain tier. One agent will be upgraded when a vacancy is produced on the next tier. In general, the probability rate ${ }^{3}$ of occurring one vacancy at a certain tier will be constant. In fact, it is easy to show that when the dying rate is constant, the number of deaths of agents in higher tiers than a certain tiers follow a Poison process, with constant probability rates. We denote $q_{n}$ the probability rate of occurring a vacancy due an upgrading in the tier $n$. The condition of replacing all the people who leave the tier is given by

$$
0=\dot{Q}_{n}=-\overbrace{d \cdot Q_{n}}^{\operatorname{die}_{n}}-\overbrace{q_{n} \cdot Q_{n}}^{\operatorname{upg}_{n}}+\overbrace{q_{n-1} \cdot Q_{n-1}}^{\operatorname{upg}_{n-1}},
$$

This condition says that, in order to maintain the number of agents in every tier $n$, the number of agents who are upgraded from the tier $n$ to the tier $n+1$ plus the number of agents who die in this tier must be the same that the number of agents who are upgraded from the tier $n-1$ to the tier $n$. Note that only the agents in the tier just below can occupy the vacancy, and not agents in lower tiers or upper tiers.

From the previous equation we can express the upgrading rate of a certain tier with respect the upgrading rate of the following tier. Then we obtain the following expression

$$
q_{n-1}=\frac{\left(d+q_{n}\right) \cdot Q_{n}}{Q_{n-1}}
$$

When the structure of the firm is finite with $N$ tiers, the upgrading probability rate of the top tier, $q_{N}$, must be 0 . For a given structure of the firm. i,e. for given values of $Q_{n}$, and a probability rate of dying $d$, the relation (2.1) establishes a recurrence for the upgrading rates that can be solved analytically. Then, for every $n<N$, we can obtain the following expression for $q_{n}$ :

${ }^{3}$ As in the process of dying, the relevant concept is the probability rate and not the probability itself. It is due we are considering the probability of being update per unity of time. 


$$
q_{n}=\frac{d \cdot \sum_{i=n+1}^{N} Q_{i}}{Q_{n}} .
$$

Let's now interpret (2.2). If we rearrange this equation we obtain, multiplying both sides by a small period of time $\Delta t$, the following equation

$$
\Delta t \cdot Q_{n} \cdot q_{n}=\Delta t \cdot d \cdot \sum_{i=n+1}^{N} Q_{i} .
$$

The left side of this equation is the number of people who is upgraded from the tier $n$ in a small period of time $\Delta t$. In the right side we have the people in higher tiers that die in this period of time $\Delta t$. In order to supply sufficiently people to preserve the number of agents in each tier, the number of upgrades in a certain tier must equal the number of deaths in all the higher tiers.

Although the agents are identical they can supply different efforts. We will treat the process of upgrading when there is a vacancy in a certain tier as a lottery among all the agents in the lower tier. We assume that the probability of winning this lottery and then be upgraded may be different for the various agents in the tier, depending on the effort individually supplied and on the distribution of efforts supplied by the rest of the agents in the tier, that every agent take as given. Then agents know that supplying effort does not guarantee to be upgraded when there is a vacancy, but alters the probability of being upgraded. This implies that the process is not properly a game, because the strategies of the individual players are not mutually influenced. Nevertheless, the distribution of strategies of the other agents in the same tier will affect the strategy of an agent, and then some concepts of the Game Theory would be considered.

The literature introduces this kind of lotteries in different ways. The optimal contract theory usually considers that the results of the work of the agents depend on the effort supplied by them in a stochastic way. When the success of the agents in their work is included in the upgrading criteria, the process is clearly dependent on the effort. Moreover, when the upgrading criteria may include the experience, the business network and other aspects that depend on the effort in a stochastic way, the process of selecting candidates to be upgraded depends stochastically on the effort individually supplied. The rank-tournaments literature is another important example of the use of effort-dependent lotteries in order to incentive the effort of the agents.

The probability rate of being upgraded of every agent individual agent in the tier depends on his or her own effort, but also on the effort supplied by the other agents 
in the same tier. Then, given a distribution of efforts of the other agents in the same tier $\tilde{\lambda}_{n}$, we note the individual probability rate of being upgraded of an agent that supplies an effort $\lambda^{i}$ as $q_{n}^{i}\left(\lambda^{i}, \tilde{\lambda}_{n}\right)$. The mean of the probabilities of being upgraded of all the agents must be equal to the probability of any agent of being upgraded, and then we have the following condition

$$
\overline{q_{n}^{i}\left(\lambda^{i}, \tilde{\lambda}_{n}\right)}=q_{n}
$$

where the upper bar means average among the agents in the tier $n$.

In some parts of this paper we will consider the simplifying assumption that the individual probability rate of being upgraded depends on the distribution of efforts of the other agents in the same tier only through its mean, $\bar{\lambda}_{n}$. It is easy to show that the only function that depends on the own individual effort and the average effort that verifies the property (2.4) for all possible realizations of $\tilde{\lambda}_{n}$ is the linear probability rate given by

$$
q_{n}^{i}\left(\lambda^{i}, \bar{\lambda}_{n}\right)=\frac{a\left(\bar{\lambda}_{n}\right)+b\left(\bar{\lambda}_{n}\right) \cdot \lambda^{i}}{a\left(\bar{\lambda}_{n}\right)+b\left(\bar{\lambda}_{n}\right) \cdot \bar{\lambda}_{n}} \cdot q_{n},
$$

with $a\left(\bar{\lambda}_{n}\right) \geq 0$. The case $b(\cdot)=0$ is the case when the probability of being upgraded is independent of the effort the agents perform. An additional possible requirement is impose $\frac{\partial}{\partial \lambda^{i}} q_{n}^{i}\left(\lambda^{i}, \bar{\lambda}_{n}\right)>0$, that is equivalent to impose that the probability of being upgraded rises with the effort, what implies $b(\cdot)>0$. In this case the probability rate of being upgraded becomes

$$
q_{n}^{i}\left(\lambda^{i}, \bar{\lambda}_{n}\right)=\frac{c\left(\bar{\lambda}_{n}\right)+\lambda^{i}}{c\left(\bar{\lambda}_{n}\right)+\bar{\lambda}_{n}} \cdot q_{n}, \text { with } c\left(\bar{\lambda}_{n}\right)=\frac{a\left(\bar{\lambda}_{n}\right)}{b\left(\bar{\lambda}_{n}\right)} \geq 0
$$

In general, under these assumptions, the probability rate of being upgraded when the effort is 0 is given by $\frac{c\left(\bar{\lambda}_{n}\right)}{\bar{\lambda}_{n}+c\left(\bar{\lambda}_{n}\right)} \cdot q_{n}$.

An interesting concrete form for the function $c(\cdot)$ is the linear form $c(s)=c \cdot s$, with $c$ a non negative constant. The derivative of the probability of being upgraded with 
respect the effort is a decreasing function of $c$, an then we can interpret $c$ as a measure of the insensibility of the premium of being upgraded. In fact, the derivative of the individual probability of being upgraded with respect the effort individually supplied is $\frac{\bar{\lambda}_{n}^{-1}}{1+c} \cdot q_{n}$. The larger $c$ is, the lesser sensible is the probability rate of being upgraded $q_{n}^{i}\left(\lambda^{i}, \bar{\lambda}_{n}\right)$ to changes in the effort individually supplied, $\lambda^{i}$. Moreover, when $c=0$ the probability of being upgraded of an agent that supplies a nil effort is 0 , and in general is $\frac{c}{1+c} \cdot q_{n}$, that is a decreasing function of $c$.

\subsection{Utility and moral hazard}

As we have seen, the firm owner pays the same wage to all the agents in a given tier. On one hand, since the effort of the agents is not observable and they do not like to supply effort, they have incentives to not supply any effort. On the other hand, because the probability of being upgraded rises with the effort supplied, if the expected utility of the agents in the next tier is higher than the expected utility of the own tier, they have incentives to supply effort in order to be upgraded and improve their level of utility. Then, the agents solve their maximization problem of deciding the effort they supply taking as given the wages of all the tiers, the structure of the firm, the utility of the next tier and the effort supplied by the other agents in the tier.

The agents being contracted have preferences on the effort they supply and the wage they earn. The agents, like in the Ramsey model (1928), want to maximize a intertemporal utility along their lives, maybe sacrificing the utility of today in order to improve the utility of tomorrow. Because agents do not know the exact moment of time they will dead, there is a discount term $e^{-d \cdot t}$, noting the probability of being alive in the time $t$. Then, when they make decisions, they make expectations about their expected utility, and then decide. An agent with an expected ${ }^{4}$ wage $w(t)$ and effort $\lambda^{i}(t)$, for each $t \in R^{+}$, has an expected utility given by

${ }^{4}$ Expected in the sense depends stochastically on the effort supplied but also in the sense that the agents do not know when they will die, and then, although the expected wage is defined for all times $t \in R^{+}$, the wage will not be earned and the effort will not supplied after their death. 


$$
U\left(w, \lambda^{i}\right)=\int_{0}^{\infty} e^{-d \cdot t} \cdot u\left(w(t), \lambda^{i}(t)\right) \cdot d t
$$

where $u\left(w, \lambda^{i}\right)$ is the instantaneous utility when the wage is and the effort is $\lambda^{i}$. As usual we will assume that $u_{w}>0, u_{\lambda}<0, u_{w w}<0$ and $u_{\lambda \lambda}<0$.

The agents will decide their effort along their life, $\lambda^{i}(t)$, and this decision will imply an expected wage in every time $t$, given by $w(t)$, because the effort of the other agents are taken as given. In general, the wage along the life of an agent, or at least his expected wage at every moment of time, will be a function of his or her effort. Then, we can suppose that agents have their expectations about the wage at every moment, $w\left(t, \lambda^{i}\right)$. The agents problem is to solve the following problem

$$
U=\max _{\lambda i} \int_{0}^{\infty} e^{-d \cdot t} \cdot u\left(w(t), \lambda^{i}(t)\right) \cdot d t
$$

with a given initial wage $w\left(0, \lambda^{i}\right)$. We will define $U_{n}$ as the maximum utility of the people who at time $t=0$ are in the tier $n$, and then have as initial wage $w\left(0, \lambda^{i}\right)=w_{n}$. The expected utility we have just presented depends only on the initial tier of the agent, that establishes the initial value of the wage. The maximizer agent solves at every moment of time the same problem of choosing the effort he or she supplies depending on the tier he or she belongs. Then we can assume that every individual agent supplies a constant effort when he or she is in a given tier, that can be different of the effort of the other agents in the same tier. Moreover, the agents in the same tier are indistinguishable, in the sense that the decisions are taken in the basis of the future, and at every moment all the agents have the same possible decisions to take. Because the agents maximize their expected utilities, they will choose the maximum expected utility, and then the expected utility of every agent of the same tier will be the same, given by $U_{n}$.

Suppose now that the expected utility of a certain tier $n+1, U_{n+1}$, is given and known. Then people who are in the lower tier faces the following maximization problem

$$
\begin{gathered}
U_{n}=\max _{\lambda i}\left[\int_{0}^{\infty} q_{n}^{i}\left(\lambda^{i}, \tilde{\lambda}_{n}\right) \cdot e^{-q_{n}^{i}\left(\lambda^{i}, \tilde{\lambda}_{n}\right) \cdot t} \cdot\left(\int_{0}^{\infty} e^{-d \cdot s} \cdot u\left(w_{n}, \lambda^{i}\right) \cdot d s+e^{-d \cdot t} \cdot U_{n+1}\right) \cdot d t\right] \\
=\max _{\lambda i}\left[\frac{u\left(w_{n}, \lambda^{i}\right)+q_{n}^{i}\left(\lambda^{i}, \tilde{\lambda}_{n}\right) \cdot U_{n+1}}{d+q_{n}^{i}\left(\lambda^{i}, \tilde{\lambda}_{n}\right)}\right],
\end{gathered}
$$


where $\tilde{\lambda}_{n}$ is the distribution of the efforts of the agents in the tier $n$, that every single agent takes as given, and $q_{n}^{i}\left(\lambda^{i}, \tilde{\lambda}_{n}\right)$ is the upgrading probability rate of an agent of the tier $n$ that supplies an effort $\lambda^{i}$ when the distribution of efforts of this tier is $\tilde{\lambda}_{n}$. This formula provides us a relation among utilities of different tiers, and will be useful in the future in order to solve recurrently the problem.

The agents in the highest tier, the tier $N$, do not have any incentive to work, because they can not be upgraded. These agents do not supply any effort, and only leave this tier when they die. The utility of the agents in the tier $N$ is given by

$$
U_{N}=\frac{u\left(w_{n}, 0\right)}{d} .
$$

This formula can be used, through the equation (2.6), to obtain $U_{N-1}$, and then obtain $U_{N-2}, \ldots$ Then, we can obtain all the utilities from the top tier to the bottom.

Using the expression for the expected utility (2.6) we have that the incentive compatibility condition can be written, for an agent belonging to the tier $n$ that supplies an effort $\lambda^{i}$ and for an effort distribution on the tier $\tilde{\lambda}_{n}$, in the following form:

$$
\frac{u\left(w_{n}, \lambda^{i}\right)+q_{n}^{i}\left(\lambda^{i}, \tilde{\lambda}_{n}\right) \cdot U_{n+1}}{d+q_{n}^{i}\left(\lambda^{i}, \tilde{\lambda}_{n}\right)} \begin{cases}=\frac{u\left(w_{n}, \lambda^{i}\right)+q_{n}^{i}\left(\lambda^{i}, \tilde{\lambda}_{n}\right) \cdot U_{n+1}}{d+q_{n}^{i}\left(\lambda^{i}, \tilde{\lambda}_{n}\right)} & \text { if } \lambda^{\prime i} \in \tilde{\lambda}_{n} \\ \geq \frac{u\left(w_{n}, \lambda^{i}\right)+q_{n}^{i}\left(\lambda^{i}, \tilde{\lambda}_{n}\right) \cdot U_{n+1}}{d+q_{n}^{i}\left(\lambda^{i}, \tilde{\lambda}_{n}\right)} & \text { if } \lambda^{\prime i} \notin \tilde{\lambda}_{n}\end{cases}
$$

This expression says that if there is an agent that chooses $\lambda^{i}$ and another agent chooses $\lambda^{\prime i}$, their expected utility must be the same.

A stable state in our problem will be given by a set of $\left\{w_{n}, \tilde{\lambda}_{n}, Q_{n}\right\}$ for $n=1, \ldots, N$ that verifies the equations (2.6) and (2.8). In this case, by the incentive compatibility condition, the agents do not have incentives to alter the effort individually supplied in any tier, taking as given the distribution of efforts of the other agents in the same tier, $\tilde{\lambda}_{n}$. Then, every agent faces the following problem 


$$
\begin{aligned}
& 0=\frac{\partial U\left(w_{n}, \lambda^{i}\right)}{\partial \lambda^{i}}=\frac{\partial}{\partial \lambda^{i}}\left(\frac{u\left(w_{n}, \lambda^{i}\right)+q_{n}^{i}\left(\lambda^{i}, \tilde{\lambda}_{n}\right) \cdot U_{n+1}}{d+q_{n}^{i}\left(\lambda^{i}, \tilde{\lambda}_{n}\right)}\right) \\
& =\frac{\left(d \cdot U_{n+1}-u\left(w_{n}, \lambda^{i}\right)\right) \cdot \frac{\partial}{\partial \lambda^{i}} q_{n}^{i}\left(\lambda^{i}, \tilde{\lambda}_{n}\right)+\left(d+q_{n}^{i}\left(\lambda^{i}, \tilde{\lambda}_{n}\right)\right) \cdot u_{\lambda}\left(w_{n}, \lambda^{i}\right)}{\left(d+q_{n}^{i}\left(\lambda^{i}, \tilde{\lambda}_{n}\right)\right)^{2}}
\end{aligned}
$$

Finally, this relation can be arranged in order to obtain an interpretable expression for the incentive compatibility condition:

$$
\begin{gathered}
-u\left(w_{n}, \lambda^{i}\right)=\frac{\left(d \cdot U_{n+1}-u\left(w_{n}, \lambda^{i}\right)\right) \cdot \frac{\partial}{\partial \lambda^{i}} q_{n}^{i}\left(\lambda^{i}, \tilde{\lambda}_{n}\right)}{d+q_{n}^{i}\left(\lambda^{i}, \tilde{\lambda}_{n}\right)} \\
=\left(U_{n+1}-U_{n}\right) \cdot \frac{\partial}{\partial \lambda^{i}} q_{n}^{i}\left(\lambda^{i}, \tilde{\lambda}_{n}\right) .
\end{gathered}
$$

This equation means that marginal increment of expectancy gain of utility (right side) must equal the marginal disutility of increasing this quantity of effort (left side).

\section{Two-valued EFFort}

The first interesting case is the two valued effort (TVE) case, that consists in considering that the agents, in their decision of supply effort, can only choose among two given values, a low effort and a high effort. Then, in every tier there will be two subsets of agents, the agents that supply a low effort and the agents that supply a high effort. We will make the simplifying assumption that the low effort is 0 for all tiers, and the high effort value of the tier will be noted by $\lambda_{n}$.

This first simplifying assumption is common in the study of the principal-agent problems, where the agent can choose among a finite number (often only 2) possible elections unobservable by the principal. An example when this condition holds is, for example, when there is an important cooperation with the different agents who work and the nature of the work does not permit to work more than the others of the group. 
Otherwise, there are many other tasks that can be done or not, but there are no intermediate possible elections.

Let's assume a linear individual probability rate of being upgraded, as the shown in (2.5). Moreover lets assume that the function takes the linear form $c(s)=c \cdot s$, with $c$ a non negative constant. In this case, the individual probability of being upgraded for an agent being in the tier depends on the effort individually supplied $\lambda^{i}$ in the following form

$$
q_{n}^{i}\left(\lambda^{i}, \bar{\lambda}_{n}\right)= \begin{cases}\frac{c}{1+c} & \text { if } \lambda^{i}=0, \\ \frac{\lambda_{n}+c \cdot \bar{\lambda}_{n}}{\bar{\lambda}_{n} \cdot(1+c)} & \text { if } \lambda^{i}=\lambda_{n},\end{cases}
$$

were, as before, $\bar{\lambda}_{n}$ is the average effort of the tier $n$. We denote $\gamma_{n} \in[0,1]$ as the proportion of agents in the tier that supplies the higher effort $\lambda_{n}$, named working agents. This definition allows us to write the average effort of the tier $n$ as $\bar{\lambda}_{n}=\gamma_{n} \cdot \lambda_{n}$. When $\gamma_{n}=0$ the production of the agents in the tier $n$ will be 0 , as in the tier $N$, where we always have $\gamma_{N}=0$. When $\gamma_{n}=1$ all the agents in the tier $n$ supply the high effort.

The problem that an agent of the tier $n$ faces when he or she decides the effort supplied when the utility of the next tier $U_{n+1}$ is given is the following maximization problem:

$$
\left.\begin{array}{c}
U_{n}=\max _{\left.\lambda \in \in 0, \lambda_{n}\right\}}\left\{\frac{u\left(w_{n}, \lambda^{i}\right)+\frac{c \cdot \bar{\lambda}_{n}+\lambda^{i}}{\bar{\lambda}_{n} \cdot(1+c)} \cdot q_{n} \cdot U_{n+1}}{d+\frac{c \cdot \bar{\lambda}_{n}+\lambda^{i}}{\bar{\lambda}_{n} \cdot(1+c)} \cdot q_{n}}\right\} \\
=\max \left\{\frac{u\left(w_{n}, 0\right)+\frac{c}{1+c} \cdot q_{n} \cdot U_{n+1}}{d+\frac{c}{1+c} \cdot q_{n}}, \frac{u\left(w_{n}, \lambda_{n}\right)+\frac{1+c \cdot \gamma_{n}}{\gamma_{n} \cdot(1+c)} \cdot q_{n} \cdot U_{n+1}}{1+c \cdot \gamma_{n}} \cdot q_{n}\right.
\end{array}\right\}
$$


The agents will choose the level of effort that maximizes their utility. Let's first consider that the equilibrium is reached when $0<\gamma_{n}<1$, that is, when the two options (supplying a low or a high effort) have the same expected utility. In this case the following condition holds

$$
\frac{u\left(w_{n}, 0\right)+\frac{c}{1+c} \cdot q_{n} \cdot U_{n+1}}{d+\frac{c}{1+c} \cdot q_{n}}=U_{n}=\frac{u\left(w_{n}, \lambda_{n}\right)+\frac{1+c \cdot \gamma_{n}}{\gamma_{n} \cdot(1+c)} \cdot q_{n} \cdot U_{n+1}}{d+\frac{1+c \cdot \gamma_{n}}{\gamma_{n} \cdot(1+c)} \cdot q_{n}}
$$

The left side of the equation allows us to find a relation among the expected utility of every tier and the expected utilities of the next tiers. Using the formula (2.7) we can then obtain the expected utility of a given tier $n$ as a function of the wage of this tier and the wages of all the tiers higher than $n$. In general this is a complicated relation, except in the case when $c=0$, because in this case the utility of all tiers is given by $\frac{u\left(w_{n}, 0\right)}{d}$.

Note that the equation (3.1) may not hold when all agents work, $\gamma_{n}=1$, or when no agent works, $\gamma_{n}=0$. If, for example, all the agents in the tier $n$ work we can suppose that the maximizer capitalist, in order to maximize its profits, will lower the wage of the tier $n+1$ until the moment that the agents in the tier $n$ are indifferent to work and not work. Doing this the firm owner reduces the costs, the production of the tier $n$ is not altered and the proportion of working agents in the tier $n+1$ do not decrease, because the difference between the utilities of the tier $n+1$ (that decreases) and the tier $n+2$ (that remains constant) is higher than before. Nevertheless, lowering the wage of the tier $n+1$ agents their utility also decrease, and then the utility of the tier $n$. This fact can lower the average effort supplied by the agents in the lower tiers and then decrease the profits. In the case $n=1$, because changes in the utility of this tier do not affect at the utility of other tiers, and then the equation (3.1) even in the case $\gamma_{1}=1$.

When the equation (3.1) holds we can obtain the formula for the proportion of working agents, given by 


$$
\gamma_{n}=\frac{\bar{\lambda}_{n}}{\lambda_{n}}=\frac{q_{n} \cdot\left(U_{n+1}-\frac{u\left(w_{n}, 0\right)}{d}\right)}{d \cdot\left((1+c) \cdot d+c \cdot q_{n}\right) \cdot\left(u\left(w_{n}, 0\right)-u\left(w_{n}, \lambda_{n}\right)\right)} .
$$

In the numerator of this expression we can interpret $\frac{u\left(w_{n}, 0\right)}{d}$ as the expected utility of an agent that remains all his or her life in the firm in the tier $n$ without supplying effort. This will occur, for example, when $c=0$, because in this case the agent that does not supply effort remains always in the same tier until his or her death. The proportion of workers that supply the high effort is 0 only when the expected utility of the next tier, $U_{n+1}$, is equal to the expected utility in the case if the agent remains until his or her death in the tier $n$ without supplying any effort. It may be surprising that this result not only holds when $c=0$, that is, when supplying a nil effort implies the impossibility of being upgraded, but also when an agent that supplies a 0 effort can be upgraded.

We observe from (3.2) that the number of agents that supply the high effort depends differently on the various variables. It depends positively on the probability rate of being upgraded, because more possibilities of upgrading the agents have, more incentives to supply effort they have. It depends negatively on the death rate, because less important is the future (higher death rate), less incentives they have to supply effort. It depends positively on the difference of the utility of the next tier and the current tier, because larger is the difference of utilities, more incentives the agents have to supply effort. It depends negatively on the difference of the utility of non making effort and making effort, because larger is the difference or utility on working or non working, more incentives have the agents to not supply effort.

Let's measure the profits that a firm faces for a given set of wages $\left\{w_{n}, n=1, \ldots, N\right\}$. Using (3.2) we obtain the following formula for the profits

$$
\begin{gathered}
P^{n o m}=\sum_{n=1}^{N-1} p \cdot A_{n} \cdot \lambda_{n} \cdot \gamma_{n} \cdot Q_{n}-\sum_{n=1}^{N-1} w_{n} \cdot Q_{n} \\
=\sum_{n=1}^{N-1}\left(\frac{p \cdot A_{n} \cdot \lambda_{n} \cdot \gamma_{n} \cdot q_{n} \cdot\left(d \cdot U_{n+1}-u\left(w_{n}, 0\right)\right)}{((1+c) \cdot d+c \cdot q) \cdot\left(u\left(w_{n}, 0\right)-\left(u\left(w_{n}, \lambda_{n}\right)\right)\right.}-w_{n}\right) \cdot Q_{n} .
\end{gathered}
$$


If the efforts $\left\{\lambda_{n}, n=1, \ldots, N-1\right\}$ can not be chosen by the firm owner, the firm faces the problem choose the set of wages that maximize the profits. Then, the first order condition (FOC) is a set of $N$ differential equations with the following structure

$$
0=\frac{\partial P^{n o m}}{\partial w_{n}}, \quad n=1, \ldots, N .
$$

This set of equations is in general hard to resolve because we have $N$ entwined equations with $N$ unknown variables. Moreover, when the firm owner can choose the high effort of every tier, $\lambda_{n}$ for $n=1, \ldots, N$, we should add $N-1$ equations to the equations system, since the effort $\lambda_{N}$ is irrelevant because all agents in the tier $N$ supply an effort equal to 0 .

Let's now add to our model some simplifying assumptions that will allow us to obtain analytical results. These assumptions are not too restrictive and they will allow us to obtain new interesting general results, that will be useful to interpret the firm structure in depth. We first assume that the utility can be decomposed in two additive parts, one depending (positively) on the wage and the other (negatively) on the effort. Then, the utility is given by

$$
u(w, \lambda)=u^{w}(w)-u^{\lambda}(\lambda)=w^{\alpha}-B \cdot \lambda^{\beta}
$$

that verify $u^{\prime w}>0, u^{\prime \lambda}>0, u^{\prime \prime w}<0$ and $u^{\prime \prime \lambda}<0$. In the particular form we have $0<\alpha<1, B>0$ and $\beta>1$. In this case is easy to obtain that $\frac{\partial \gamma_{n}}{\partial w_{n}}<0$, that is, higher is the wage of a certain tier, lower are the incentives of the agents to supply effort. When $c=0$ the profits that faces the firm can be easily written as a function of the maximizing wages of the different wage levels in the following way

$$
P^{n o m}=\sum_{n=1}^{N} w_{n} \cdot Q_{n} \cdot\left(1-\alpha \frac{w_{1}^{\alpha}}{w_{n}^{\alpha}}\right)
$$

where $w_{n}=w_{n}\left(\lambda_{1}, \ldots, \lambda_{N-1}\right)$.

Assume that the firm owner can choose the efforts $\lambda_{n}$, for $n=1, \ldots, N-1$. The maximization problem with respect to the efforts is equivalent to the following FOCs: 


$$
\begin{aligned}
& \frac{\partial P^{n o m}}{\partial w_{n}}=\frac{(1-\alpha) \cdot Q_{n}}{\alpha} \cdot\left(1-\frac{w_{1}^{\alpha}}{w_{n}^{\alpha}}\right)>0, \quad \text { for } 1<n \leq N \\
& \frac{\partial P^{n o m}}{\partial w_{1}}=-\sum_{n=1}^{N} \frac{w_{n}^{1-\alpha}}{w_{1}^{1-\alpha}} \cdot Q_{n}<0 .
\end{aligned}
$$

The first equation says that raising the wage of every tier greater than 1 the profits increase. The wages of the tiers from 2 to $N$ should be raised as possible, that is, until the moment that the proportions of working agents reach their maximum, that is, $\gamma_{n}=1$ for $n=1, \ldots, N-1$. This imply that, when the firm owner can choose the efforts, he or she chooses them providing incentives to make all the agents work. Below this tier every increase of the wage of the next tier increases the proportion of working agents, and then the production, sufficiently to compensate the increase of the costs. Over this tier an increase of the wage of the next tier does not make sense, because it does not increase the production and increases the costs.

By its hand, the wage of the first tier should be lowered as possible, that is, until $w_{1}=w_{b}$, because the derivative of the profits with respect this wage is negative. Because changes in the lowest tier wage do not affect the expected utilities of the higher tiers and then the effort supplied by the agents in the other tiers remains unchanged, when the wage is lowered the average effort of the tier 1 agents increases (an then the production) and the costs decrease. Then, when the utility is additive, the firm owner will choose the minimum possible wage for the agents in the tier 1 , $w_{1}=w_{b}$. This can not be applied to the other tiers, because changes the wage of the tier $n>1$ affect the expected utility of the agents in this tier, and then also affect the effort supplied by the agents in all the lower tiers.

Let's now consider, in order to obtain analytical results, a firm with only two tiers, assuming a general $c \geq 0$. Now the effort supplied by the second tier agents is 0 , because the second is the highest tier and its agents have no incentives to work. In this case the equation (3.1) only is applicable to $n=1$, and then this equation always holds. Using the equation (2.2) we obtain that the number of agents in the tiers 1 and 2 with respect $q_{1}$, because now we have $q_{2}=0$, are respectively $Q_{1}=\frac{d}{d+q_{1}} \cdot Q$ and $Q_{2}=\frac{q_{1}}{d+q} \cdot Q$, being the total number of workers in the firm. Because in this case the parameter $q_{1}$ determines the organization of the firm we will call it the structure parameter.

When the firm can not choose the structure parameter the only variable that the 
firm owner should decide is the wage of the second tier, noted by $w_{2}$. Using now the value of the utility of the highest tier, that is given in the equation (2.7), the equation (3.3) turns to be

$$
P^{\text {nom }}=\frac{q_{1} \cdot\left(u^{w}\left(w_{2}\right)-u^{w}\left(w_{b}\right)\right)}{\left((1+c) \cdot d+c \cdot q_{1}\right) \cdot\left(u^{\lambda}(0)-u^{\lambda}\left(\lambda_{1}\right)\right)} \cdot p \cdot A \cdot \lambda_{1} \cdot \frac{d \cdot Q}{d+q_{1}}-w_{b} \cdot \frac{d \cdot Q}{d+q_{1}}-w_{2} \cdot \frac{q_{1} \cdot Q}{d+q_{1}} .
$$

The FOC of this maximizing problem is given by the following expression

$$
u^{\prime w}\left(w_{2}\right)=\frac{q_{1} \cdot\left((1+c) \cdot d+c \cdot q_{1}\right)}{d \cdot p \cdot A \cdot \lambda} \cdot\left(u^{\lambda}\left(\lambda_{1}\right)-u^{\lambda}(0)\right)
$$

This equation that, inverting the derivative function of the wage part of the utility function, gives us the wage $w_{2}$ that maximizes the profits.

In order to obtain a concrete expression for the wage $w_{2}$, we assume a particular form for the utility form (3.4). Then, the expression that we obtain for the wage is

$$
w_{2}=\left(\frac{d \cdot p \cdot A}{\left((1+c) \cdot d+c \cdot q_{1}\right) \cdot B \cdot \lambda_{1}^{\beta-1}}\right)^{\frac{1}{1-\alpha}} .
$$

We observe from the previous relation that the wage of the 2 nd tier agents is inversely correlated to the effort. It can seem counterintuitive, because the firm owner offers high second tier wages when the high effort of the tier 1 wages is lower. But, as we will see, the variable that counterbalance this counter intuitive relation is the number of working agents. If there is a low effort and the wage of the higher tier rises, the number of working agents increases sufficiently to increase the production more than the increasing costs that comes from the rising of the wage.

If $w_{2}<w_{b}$ the wage of the tier 2 , due the condition of minimum wage, must be equal to $w_{b}$. In this case the result is the same that having a firm with only one tier, because all agents have the same wage and all agents supply a nil effort, because there are no incentives of being upgraded. The profit function is given by 


$$
P^{n o m}=Y^{n o m}-C^{n o m}=0-Q_{1} \cdot w_{b}-Q_{2} \cdot w_{2}=-Q \cdot w_{b} .
$$

Then, in this case there is no problem of optimization.

If $w_{2}>w_{b}$ then the firm owner chooses the wage $w_{2}$ given in the formula (3.6). This condition, through the inverse relation between the wage and the effort supplied, establishes a lower limit for the possible values of $\lambda_{1}$, that we call $\lambda_{b}$, where $w_{2}=w_{b}$. In the higher tier, as usual, no agent works, and in the lower tier the proportion of workers is

$$
\gamma_{1}=\frac{w_{2}^{\alpha}-w_{b}^{\alpha}}{B \cdot \lambda^{\beta}} \cdot \frac{q_{1}}{d}
$$

Using now the condition $\gamma_{1} \leq 1$ for a given $q_{1}$, we can establish a lower limit for $\lambda_{1}$, noted by $\lambda_{l}$, where $\gamma_{1}=1$, that is in general lower than $\lambda_{b}$ and that does not have an analytical form. At the same time, for a given $\lambda_{1}$, this condition establishes a upper limit for $q_{1}$.

From now we assume that $w_{2}>w_{b}$ or, what is the same, $\lambda_{1}<\lambda_{b}$. The profit where the wage of the agents in the tier 2 is chosen optimally, given by $w_{2}\left(\lambda_{1}\right)$ in (3.6), can be written in the following interesting form

$$
P^{\text {nom }}=\left(\frac{q_{1}}{d+q_{1}} \cdot \frac{(1-\alpha) \cdot w_{2}\left(\lambda_{1}\right)}{\alpha}-\frac{q_{1}}{d+q_{1}} \cdot \frac{w_{2}^{1-\alpha}\left(\lambda_{1}\right) \cdot w_{b}^{a}}{\alpha}-\frac{d}{d+q_{1}} \cdot w_{b}\right) \cdot Q
$$

were here $w_{2} \equiv w_{2}\left(\lambda_{1}\right)$. Suppose now that the firm owner can choose the effort $\lambda_{1}$, that is, the effort supplied by the working agents in the tier 1 . The FOC resulting from this maximizing problem can be expressed as:

$$
\frac{\partial P^{n o m}}{\partial \lambda_{1}}=\frac{\partial P^{n o m}}{\partial w_{2}} \cdot \frac{\partial w_{2}\left(\lambda_{1}\right)}{\partial \lambda_{1}}=\frac{(1-\alpha) \cdot q_{1} \cdot Q}{\alpha \cdot\left(d+q_{1}\right)} \cdot\left(1-\frac{w_{b}^{\alpha}}{w_{2}^{\alpha}\left(\lambda_{1}\right)}\right) \cdot \frac{\partial w_{2}\left(\lambda_{1}\right)}{\partial \lambda_{1}}<0 .
$$

Since under our assumptions this expression is always negative, the maximum profit is obtained when $\lambda_{1}$ reaches its minimum, that is, when $\lambda_{1}=\lambda_{l}$. When this condition holds, as we have seen, all tier 1 agents work, and then $\gamma_{1}=1$. It may seem surprising that the firm maximizes its profits when the effort supplied by the tier 1 agents 
is minimum and the wage of the second tier agents is maximum. As we have seen, it is due to the fact that the increase of the number of working agents more than compensates the losing of productivity per working agent and the costs of paying higher wages.

From (3.6) the effort of the working agents is inversely related with the wage of the second tier. Then, for a given $w_{b}$, the larger will be $w_{2}$, the larger will be the utility of the working agents in tier $1^{5}$, and the larger will be the utility of the agents in the tier 2. When firms maximize the profits, the result is Pareto efficient ${ }^{6}$. From this condition we can obtain the expression of $w_{b}$ with respect $w_{2}$, because the relation $w_{2}\left(w_{b}\right)$ it is not obtainable analytically. The result obtained is

$$
w_{b}=w_{2} \cdot\left(1-\left(\frac{d^{\beta} \cdot \alpha^{\beta} \cdot p^{\beta} \cdot A^{\beta}}{q_{1}^{\beta-1} \cdot\left((1+c) \cdot(d+q)-q_{1}\right) \cdot B \cdot w_{2}^{\beta-\alpha}}\right)^{\frac{1}{\beta-1}}\right)^{\frac{1}{\alpha}}
$$

We can introduce now the relative wage among the tiers in order to express analytically our variables, denoting $r_{w}=\frac{w_{b}}{w_{2}}$. The values that can take the relative wage are bounded by 0 and 1 . Then, the wage of the agents in the tier 2 takes the following form:

$$
w_{2}=\left(\frac{\alpha^{\beta} \cdot d^{\beta} \cdot p^{\beta} \cdot A^{\beta}}{B \cdot q^{\beta-1} \cdot\left((1+c) \cdot d+c \cdot q_{1}\right) \cdot\left(1-r_{w}\right)^{\beta-1}}\right)^{\frac{1}{\beta-\alpha}}
$$

Let's introduce competition among firms. In a competitive market the firms that produce the same product will compete reducing prices. Because the firm is produc-

5 The instantaneous utility of the non working agents in the tier 1 is not altered, since is given by $w_{b}^{\alpha}$, and the instantaneous utility of the working agents is increased, since the wage remains unaltered (hold in $w_{b}$ ) but the effort decreases. Nevertheless, as $\gamma_{1}$ increases, some people pass from not work to work, implying a loose of instantaneous utility.

${ }^{6}$ As is said in the footnote 5, is Pareto optimal in the sense that every «class» of agents are at least better than in any other case. In fact, the instantaneous utility of the working agents in tier 1 , the non working agents in the tier 1 and the agents in the tier 2 increase or remain constant. 
ing in real terms (quantity of good) meanwhile the workers wage is indexed nominally, when the price of the product decrease the profits decrease. Moreover, assuming that the economy is split in a large number of markets, one for every good, we can assume that the real wage of a worker do not depend on the price of the product he or she is producing, but only on the nominal wage he or she receives. This assumption is equivalent to impose that the general price level remains constant regardless the price of the good the firm produces, because it represents a small share of the consumption.

Imposing the condition of zero profits to the equation (3.3), we obtain the following relation among the final relative wage and the structure of the firm:

$$
\frac{q_{1}}{d}=\frac{\alpha \cdot r_{w}}{1-\alpha-r_{w}^{\alpha}} .
$$

This equation establishes an increasing relation between the relative wage $r_{w}$ and the firm structure parameter $q_{1}$ when competition makes the profits to be 0 . This equation implicitly give us the price as a function of the structure parameter $q_{1}$, because, in fact, we can write $r_{w}\left(p, q_{1}\right)$. Moreover we find an upper limit for the relative wage $r_{w}$, given by $r_{w}^{*} \equiv(1-\alpha)^{1 / \alpha}$. This limit corresponds to $q_{1} \rightarrow \infty$ or $Q_{2} \rightarrow Q$, that is, where all agents are in the tier 2 . Note that this relation holds regardless the firm owner can choose the high effort $\lambda_{1}$.

\section{Symmetrical Nash equilibrium}

Now we assume that every agent can choose the effort he or she supplies among all the non negative reals. The agents will increase their effort only if this decision makes their expected utility to increase, following the equation (2.9). Then, the problem turns to be a game, where many players are the agents trying to choose the best strategy, the effort they supply, in order to maximize the outcome of the game, the expected utility.

Lets now consider the symmetric Nash equilibrium (SNE) of our problem, that is, a situation where all agents have individual incentives to supply the same effort. In many problems of identical players treated in the context of the Game Theory the symmetrical Nash equilibrium is a fundamental concept, which is used to obtain results of many different problems, that go from firms competition to consumer's 
behavior. In this case we can consider the competition of the agents in every tier as a game with identical players, where the strategy of every agent is given by the decision of the effort he or she supplies. The final level of effort that is common among the agents in a certain tier is neither decided by the firm owner nor the individual agents, but from a process of competition among the agents that ends with a state where all agents individually decide to supply the same effort than the other agents.

Let's return to the condition of immediate occupation of the vacancies of the first tier. In the TVE case the assumption that there is a pool of unemployed agents disposed to work in the firm at wage $w_{b}$ (established by the government) or that the agents have a reserve utility were essentially the same when $c=0$. It was due the expected utility of the agents in the tier 1 was $w_{b}^{\alpha} / d$, and then the utility and the minimum wage are clearly correlated. The SNE case can be solved assuming that the firm has to provide a minimum utility in the first tier, instead of a minimum wage, and the results are similar to those obtained with the minimum wage assumption. Nevertheless we will assume that there is a minimum wage established nominally by the government, because the analytical expressions are more simple and the results are easily comparable with the TVE case.

In this case we can use, additionally to the equation (2.6), the equation (2.9), that states that every agent, given the effort supplied by the other agents, chooses his or her effort in order to maximize the own utility. This equation shows that, when the agents choose the effort they supply among all real values, they choose a local maximum. In our case, in a symmetrical solution, all agents supply the average effort of the tier considered, that is $\lambda^{i}=\bar{\lambda}_{n}$ and $q_{n}^{i}=q_{n}$. Then, the condition (2.9) takes the following form

$$
\frac{\left.\left(d \cdot U_{n+1}-u\left(w_{n}, \bar{\lambda}_{n}\right)\right) \cdot \frac{\partial}{\partial \lambda^{i}} q_{n}^{i}\left(\lambda^{i}, \bar{\lambda}_{n}\right)\right|_{\lambda^{i}=\bar{\lambda}_{n}}}{d+q_{n}}=-u_{\lambda}\left(w_{n}, \bar{\lambda}_{n}\right) .
$$

We assume a linear function $q_{n}^{i}\left(\lambda^{i}, \bar{\lambda}_{n}\right)$ as the shown (2.5). In particular, as in the case of a two valued effort, we consider $c(s)=c \cdot s$, with a non negative constant. Given these assumptions the equation (2.9) turns to be

$$
\frac{d \cdot U_{n+1}-u\left(w_{n}, \lambda_{n}\right)}{(1+c) \cdot \lambda_{n}} \cdot q_{n}=-\left(d+q_{n}\right) \cdot u_{\lambda}\left(w_{n}, \lambda_{n}\right) .
$$

where, from now, we will denote $\lambda_{n} \equiv \bar{\lambda}_{n}$. This equation allows us to find a relation 
between $\lambda_{n}$ and $w_{n}$, depending on $U_{n+1}$. Using this relation the firm owner can maximize the profits choosing the maximizing wage for every tier.

In order to obtain analytical results we assume the same utility function as in the two valued effort case (3.4), a form with two additive terms, one depending on the effort and the other on the wage. Moreover, we will assume that the two components of the utility have a form with constant elasticity of substitution, formally the same utility that is used in the previous section.

Let's now consider, as in the case of a two-valued effort, the case of a firm with only two tiers, in order to obtain analytical results and to compare them with the obtained in the former case. Recall that the main difference between these two models is the fact that in the two valued effort case the level of effort was given or established by the firm owner while in this case the effort is decided by the competing agents $^{7}$. As in the TVE case, we have, and then the number of agents of the different tiers are given by $Q_{1}=\frac{d}{d+q_{1}} \cdot Q$ and $Q_{2}=\frac{q_{1}}{d+q_{1}} \cdot Q$. The tier 2 now is the highest tier and then the effort supplied by its agents is 0 , and then the utility of the agents in this tier is the given by (2.7).

The concrete form of the utility we have assumed allows us to express the equation (4.1) in the following way

$$
\frac{d \cdot\left(w_{2}^{\alpha}-w_{1}^{\alpha}-B \cdot \lambda_{1}^{\beta}\right)}{\lambda_{1} \cdot(1+c)} \cdot q_{1}=\left(d+q_{1}\right) \cdot B \cdot \beta \cdot \lambda_{1}^{\beta-1}
$$

Then, this maximization condition allows us to write the a relation among the effort supplied by the agents in the tier 1 and the wage of the agents in the tier 2 , that is given by

$$
\lambda_{1}=\left(\frac{q_{1} \cdot\left(w_{2}^{\alpha}-w_{1}^{\alpha}\right)}{B \cdot\left(\beta \cdot(1+c) \cdot\left(d+q_{1}\right)-q_{1}\right)}\right)^{\frac{1}{\beta}}
$$

7 Another difference is that in the two valued effort model we allow to work only a part of the agents, and the others supply effort 0 . Nevertheless, as we have seen, when the firm owner can choose the higher effort he ore she has incentives to make them all supply effort, and then the difference disappears. 
In this case, differently of the TVE case, where the effort is related to the wage through the equation (3.6), the effort supplied depends positively on the tier 2 wage. As in the case of the TVE, the firm owner had incentives to hold the wage of the agents in the lowest tier to the lowest possible wage, because, as we have just seen, lowering this wage the agents supply a higher effort (increasing the production) and the costs decrease. Then, we have that $w_{1}$ coincides with the minimum wage, $w_{1}=w_{b}$.

Suppose first that the firm organization is given, that is, the firm owner takes $q_{1}$ as given. The problem that faces the firm owner is to find the wage of the tier 2 agents that maximizes his or her profit, that is

$$
\max _{w_{2} \in R^{+}}\left[p \cdot A \cdot\left(\frac{q_{1} \cdot\left(w_{2}^{\alpha}-w_{b}^{\alpha}\right)}{B \cdot\left(\beta \cdot(1+c) \cdot\left(d+q_{1}\right)-q_{1}\right)}\right)^{\frac{1}{\beta}} \cdot \frac{d \cdot Q}{d+q_{1}}-w_{b} \cdot \frac{d \cdot Q}{d+q_{1}}-w_{2} \cdot \frac{d \cdot Q}{d+q_{1}}\right] .
$$

The corresponding FOC to this problem, as in the case of a two valued effort, is an equation impossible to solve analytically for $w_{2}$. Nevertheless, as in the case of the two valued effort, we can obtain the expression or $w_{b}$ with respect $w_{2}$, that now is given by

$$
w_{b}=w_{2} \cdot\left(1-\left(\frac{d^{\beta} \cdot \alpha^{\beta} \cdot p^{\beta} \cdot A^{\beta}}{q_{1}^{\beta-1} \cdot\left(\beta \cdot(1+c) \cdot\left(d+q_{1}\right)-q_{1}\right) \cdot \beta^{\beta} \cdot B \cdot w_{2}^{\beta-\alpha}}\right)^{\frac{1}{\beta-1}}\right)^{\frac{1}{\alpha}}
$$

This relation is similar to (3.8). The only difference is a term in the denominator of the division inside the parenthesis, that in the TVE case is $\left((1+c) \cdot\left(d+q_{1}\right)-q_{1}\right)$ and now turns to be $\left(\beta \cdot(1+c) \cdot\left(d+q_{1}\right)-q_{1}\right) \cdot \beta^{\beta}$. Note that the two results are equivalent if we replace all the $\beta$ that not appear in the exponents by 1 in (4.2).

Lets now write $w_{2}$ as a function of the relative wage. As in the TVE case, it is useful to express the quantities with respect the relative wage $r_{w}=\frac{w_{b}}{w_{2}}$. Introducing the relative wage to the equation (4.2), it can be inverted and we obtain

$$
w_{2}=\left(\frac{d^{\beta} \cdot a^{\beta} \cdot p^{\beta} \cdot A^{\beta}}{B \cdot \beta^{\beta} \cdot\left(\beta \cdot(1+c) \cdot\left(d+q_{1}\right)-q_{1}\right) \cdot\left(1-r_{w}^{\alpha}\right)^{\beta-1}}\right)^{\frac{1}{\beta-\alpha}}
$$


Then, for a given $q_{1}$ and $p$, when the relative wage is hold at some level, $w_{2}$ become automatically defined. Comparing this equation with the analogous one in the case of a two-valued effort, (3.9), we observe that the only difference is again only given by the terms that have $\beta$ outside the exponents. Note that, all things equal, $w_{2}$ is lower in the SNE case than in the TVE case.

Now consider the situation where competition among firms makes the the price decrease until the moment where firm faces zero profits. Similarly to the TVE case, we can obtain the following condition that ensures that the profits become 0 :

$$
\frac{q_{1}}{d}=\frac{\frac{\alpha}{\beta} \cdot r_{w}}{1-\frac{\alpha}{\beta}-r_{w}^{\alpha}} .
$$

This equation is another time similar to the same condition in the TVE case, shown in (3.10), being equal when we replace $\alpha$ by $\alpha / \beta$, except in the exponent, in this equation. Then, the upper limit for the relative wage is given by $(1-\alpha / \beta)^{1 / \alpha}$. This point establishes the upper bound of $r_{w}$, and corresponds to the configuration where all agents are in the tier 2. Given the equation (4.4) we can express all the variables of our problem with respect the relative wage or the structure parameter $q_{1}$.

\section{COMPARISON}

In this section we try to compare the results obtained in the TVE case and the SNE case, presented in the sections 3 and 4 . The two cases we have treated present a lot of similarities and few differences, but small differences in the short run can produce huge differences in long run.

In both cases we have studied previously only one variable remains unconstrained, once the maximization problem is solved and the condition of nil profits holds, and when the firm organization can be chosen by the firm owner. For convenience, to obtain analytical expressions for all the variables, in each case we have chosen the relative wage $r_{w}$ to be the free variable. Nevertheless, using this variable is difficult to compare the two cases presented, because the range of the possible relative wages are different, form 0 to $(1-\alpha / \beta)^{1 / \alpha}$ in the case of the two valued effort and from 0 to $(1-\alpha / \beta)^{1 / \alpha}$ in the case in the two groups, as it can be seen in the expres- 
sions (3.10) and (4.4). Then we need another variable to compare the figures of the two models.

We can use as variable by representing the number of agents in the tier 2, given by $Q_{2}=\frac{q_{1}}{q_{1}+d} \cdot Q$, that goes from 0 (all agents in the tier 1 ) to $Q$ (all agents in the tier 2). Remember that in both cases the relations (3.10) and (4.4) establish a strictly increasing function of the number of agents of the tier 2 and the relative wage. Then, the number of agents in the tier 2 is a good variable, because determines all other variables in one way and has the same range in both cases. The number of agents in the tier 2 does not allow us to write analytical expressions, but allows us to compare graphically the different relevant variables. Moreover, the proportion of agents among the two tiers many times is given by specificities of the productive process of every concrete good, what makes this variable more suitable when we analyze a concrete good.

\subsection{At firm level}

The firm takes the price of the good produced and the nominal minimum wage of the economy (both indexed in monetary units and not in product units) as given. As we have seen, then it maximizes its profits choosing the optimal wage of the second tier workers, and, in the TVE case, the high effort of the tier 1 agents.

In order to compare the results obtained for the TVE case and the SNE case lets first look at the figure 1 (a), that corresponds to the wages and efforts in a single firm for every given quantity of agents in the tier 2, when at every point the price verifies the free entry condition, implying nil profits, that depends on the structure of the firm. We have assumed that the nominal minimum wage $w_{b}$ is established exogenously. At a single firm level the nominal wages coincide with the real wages, because we assume that the good that the firm produces represents a small part of the consumption of the agents, and then changes in its price do not alter the general price level. Then, the workers of a firm consider the nominal wages when they decide the effort supplied. We observe that both the effort and the second tier wage are higher in the TVE case. 
Figure 1. In (a) we represent the nominal wages and the efforts in a single firm for the TVE case (in black) and the SNE case (in gray). In (b) are depicted the nominal and real productions of a single firm and the price when profits are 0, the TVE case in black and the SNE case in gray

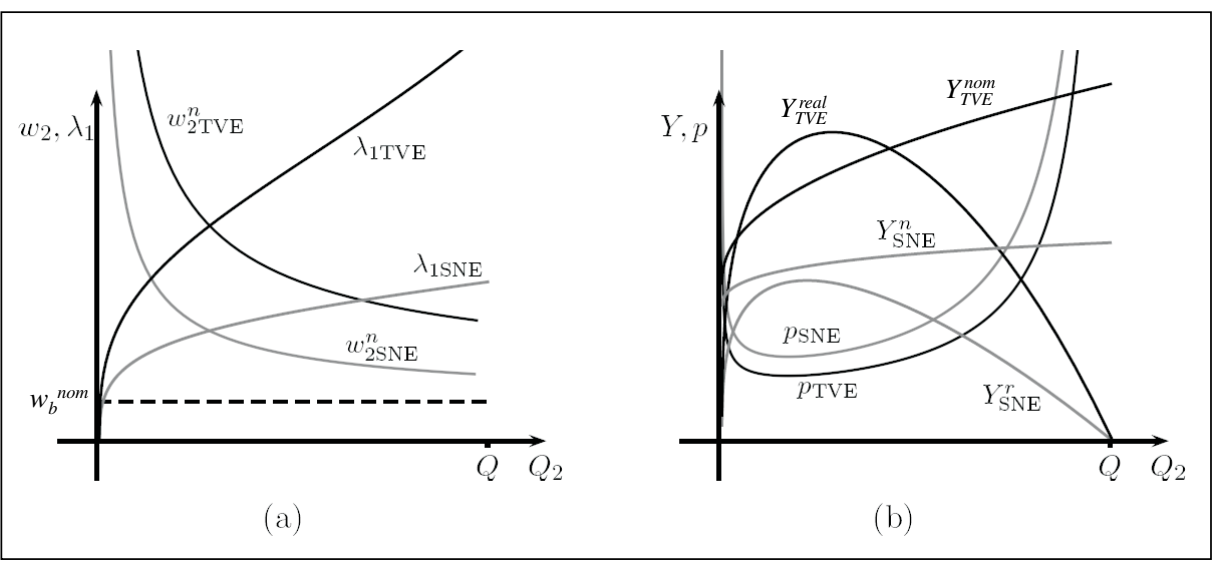

If one sector face extraordinary profits, new firms are attracted to the sector, and then the price decreases, until the moment that profits are 0 . In the figure 1 (b) we represent the nominal production, the real production (measured in number of units produced) and the price of the product that implies nil profits for all possible structures of the firm, parameterized by $Q_{2}$. Every sector in the economy may have specificities in the production process that establish a concrete $Q_{2}$, and then the price of the product will tend to the value of the price depicted. Our model predicts differences of prices among different goods due the differences in the organizations of the firm that produce them. Cause, as we have just seen in the part (a) of this figure, the effort supplied by the tier 1 agents is higher in the TVE case, and so is the real production in this case. Moreover, because the nominal wage paid to the tier 2 agents in the TVE case is higher than in the SNE case, it implies that the nominal production in the TVE case must be higher than in the SNE case.

When the structure parameter can not be eligible by the firms, the competition decreases the price until the moment that the profits turn to be 0 . The price when the profits are 0 takes the following form:

$$
p=\frac{w_{b} \cdot d+w_{2} \cdot q_{1}}{\lambda_{1} \cdot A \cdot d}=\frac{w_{b}}{\lambda_{1} \cdot A}+\frac{w_{2} \cdot q_{1}}{\lambda_{1} \cdot A \cdot d}
$$


This price function $p\left(q_{1}\right)$ is a $\mathrm{U}$-shaped function, considering $\lambda_{1}=\lambda_{1}\left(q_{1}\right)$ and $w_{2}=w_{2}\left(q_{1}\right)$, and then it has an interior minimum. We observe in the figure figure 1 (b) that the 0 profits price is higher in he SNE case, making the firms less competitive.

When firms can choose the tier structure parameter, firms tend to choose near the structure that lowers the price in order to be more competitive and then obtain additional profits. This process ends when the price reaches its minimum. Then, the competitive market makes the firms choose a structure that verifies the condition $\partial p / \partial q_{1}=0$. The nominal production is a increasing function of $q_{1}$, but not the real production production, that is a hill-shaped function. Note that the maximum of the real production does not coincide with the minimum price.

\subsection{At the whole economy level}

When we consider the whole economy, we aggregate the production of all the firms and the money becomes neutral. Once the nominal minimum wage is established by the government, the price is set up by the competition among firms, and then the real minimum wage is determined. Intuitively, at an aggregated level we can consider that only one good is produced, and then the agents are payed in real terms with respect to this good.

We consider now the price of the aggregated production as the price level of the economy. Because the agents will make the decision of choosing the effort supplied taking in account the real wages of every tier, let's traduce the nominal wages to the real wages. At the whole economy level the real wages are obtained by dividing the nominal wages by the price, that now coincides with the general price level. We have that the nominal minimum wage is held exogenously and the real minimum wage is $w_{b}^{\text {real }}=w_{b}^{\text {nom }} / p$, the maximum of the real minimum wage must coincide with the minimum of the price. As we said, when the structure parameter is eligible by the firms, the competition moves the economy to the minimum of the price function, that coincides with the maximum of the real minimum wage. In our problem, the free market maximizes the purchasing power of the less well payed workers.

In the figure 2 (a) we observe the different real wages and the consequent efforts reached when the whole economy is considered. Because the 0 profits price was higher in the SNE case than in the TVE case and the nominal minimum wage is held, in the whole economy the SNE real minimum wage will be lower than the TVE real minimum wage. This fact, together with the fact that relation between the nominal 
wage of the tier 2 agents and the nominal minimum wage is higher in the TVE case, establishes that the real wage of the agents in the tier 2 is high in the TVE case. Finally, because when the whole economy is considered all the real wages tend to 0 when $Q_{2}$ tends to $Q$, the effort supplied tends to 0 in this limit, being higher in the TVE case in order to produce enough to pay higher real wages.

Figure 2. In (a) are depicted real wages and the efforts when the whole economy is considered for the TVE case (in black) and the SNE case (in gray). In (b) are depicted the average instantaneous utility in a single firm case (noted with «sf») and when the whole economy is considered (noted with «we»), the TVE case in black and the SNE case in gray

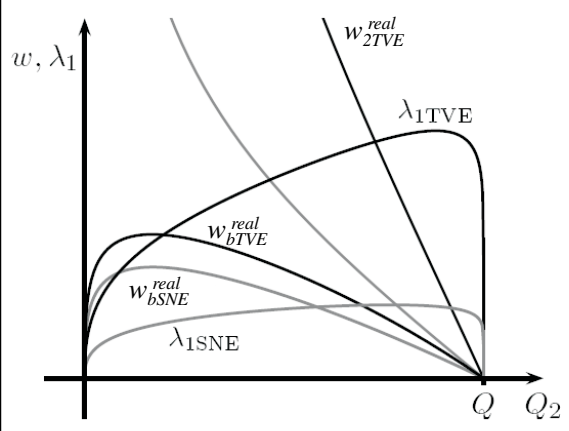

(a)

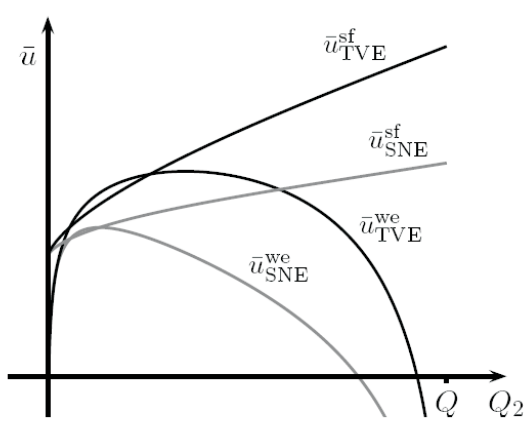

(b)

As we said before the whole economy will tend to be in the minimum of the price, that coincides with the maximum of the real minimum wage. In this point the instantaneous utility of the tier 2 agents decreases with $Q_{2}$, because $w_{2}^{\text {real }}$ decreases with $Q_{2}$, and because the effort of the tier 1 agents increases with $Q_{2}$, the instantaneous utility of the tier 1 agents is also a decreasing function of $Q_{2}$. Then, this point may seem non Pareto efficient, in the sense that lowering $Q_{2}$ we increase the instantaneous utility of both the tier 1 agents and the tier 2 agents, and then we make everyone better off. Nevertheless, the utility that the agents consider when they make decisions is not the instantaneous utility, but the expected utility.

From (2.6) we can obtain the form of the expected utilities of the first tier workers: 


$$
\begin{aligned}
U_{1}= & \frac{\frac{u\left(w_{1}, \lambda_{1}\right)}{d}+q_{1} \cdot U_{2}}{d+q_{1}}=\frac{u\left(w_{1}, \lambda_{1}\right)}{d} \cdot \frac{d}{d+q_{1}}+\frac{u\left(w_{2}, 0\right)}{d} \cdot \frac{q_{1}}{d+q_{1}} \\
= & \frac{u\left(w_{1}, \lambda_{1}\right)}{d} \cdot \frac{Q_{1}}{Q}+\frac{u\left(w_{2}, 0\right)}{d} \cdot \frac{Q_{2}}{Q}=\frac{\bar{u}}{d}
\end{aligned}
$$

where we have used the fact that the all the agents in the tier 1 supply effort both in the TVE case and in the SNE case. The expected utility of the workers that belong to the firm in the tier $1, U_{1}$, coincides with the average instantaneous utility of the economy, $\bar{u}$, divided by the death rate $d$. Note that when the instantaneous utilities of all the agents in the economy decrease with $Q_{2}$, the increase of the number of agents in the tier 2 (with high instantaneous utility) can compensate the decrease of the instantaneous utilities, and make the average utility rise.

It is interesting to note that when $c=0$ in the TVE case, that implies that the tier 1 agents that do not supply effort have a nil probability rate of being upgraded, the expected utility of the agents in the tier 1 takes the simple form $U_{1}=w_{b}^{\alpha} / d$. In this case we have that the market economy, when the structure of the firms is eligible, will tend to maximize the expected utility of the agents in the tier 1, and, as we have just seen, also the average instantaneous utility will be maximized. Then, the competitive market allows the economy to reach the aggregated social optimum.

The figure 2 (b) represents the different average utilities in the single firm and in the whole economy depending on $Q_{2}$. Like nominal and real productions, the average utilities behave very different in a single firm and at the whole economy level. In a single firm the average utility is a increasing function of $Q_{2}$, like the nominal production, while the average utility when the whole economy is considered is a hillshaped function, like the real production. Although in the TVE case the effort is higher than in the SNE case, the higher nominal and real wages make the utilities be higher than the SNE case, being then the TVE case strictly preferred than the SNE case.

An important consequence of the figure 2 (b) is that the firms tend to prefer to have a regulated structure defined by the government, with $Q_{2}$ as higher as possible. As we have seen in the figure $1(\mathrm{~b})$, when $Q_{2}$ is fixed high the price and the nominal production are high and, what is more important, the average utility of the members of this sector is high. But if it is applied to the whole economy, the price level increases and the nominal wages decrease, making the whole average utility decrease. It is 
an example of the fact that what is better for every part of the economy (a regulation with high $Q_{2}$ for a sector) can be worse for the whole economy.

As we have seen, the TVE case is more effective than the SNE case, because in this case the production and the real wages are higher than in the SNE case. It is due because in the TVE case the agents have a small set of eligible efforts (in fact, they have only two options compared with all the reals in the SNE case). In the TVE case the worker can only compare two cases, meanwhile in the SNE case the effort must be a local optimum. This fact allows to obtain a higher average effort, and then higher production, and then real wages in the TVE case. Moreover, although the effort is higher in the TVE case, it has a higher average instantaneous utility, and then it is also preferable at a social aggregated level.

\subsection{The Gini index}

Let's now look at the inequality produced in this model, and the information that gives us the Gini index. We will inquire about the existence of the classical tradeoff between efficiency and equality, and we will analyze the consequences of choose a value for the firm organization parameter $q_{1}$, or indirectly choose a minimum wage.

Let's suppose that the firm faces total profits equal to 0 . In order to calculate Gini index note that the total income is given by $Q_{1} \cdot w_{b}+Q_{2} \cdot w_{2}$. For a given $q_{1}$ the Lorentz curve is the same in the two cases ${ }^{8}$ is given by

$$
g(\sigma)=\left\{\begin{array}{cc}
\frac{w_{b} \cdot\left(d+q_{1}\right)}{d \cdot w_{b}+q_{1} \cdot w_{2}} \cdot \sigma & \text { for } \sigma<\frac{d}{d+q_{1}}, \\
1-\frac{w_{2} \cdot\left(d+q_{1}\right)}{d \cdot w_{b}+q_{1} \cdot w_{2}} \cdot(1-\sigma) & \text { for } \sigma>\frac{d}{d+q_{1}},
\end{array}\right.
$$

${ }^{8}$ In fact, the Lorentz curve (and then the Gini index) when there are two groups of people, the Gini index only depends on the relative wage, $w_{b} / w_{2}$, and their relative populations, $d / q_{1}$. 
This curve is a composition of two straight lines in two different intervals, one corresponding to the low wage people (from 0 to $\frac{d}{d+q_{1}}$ ) and the other corresponding to the high wage people (from $\frac{d}{d+q_{1}}$ to 1 ), and is depicted in the figure 3 (a) for a given relative wage (black line) for the TVE case. For the different values of the wages the vertexes of this curve lay on the curved dashed line. As we can observe this curve takes the value $1-\alpha$ when $\sigma \rightarrow 1$, meaning that in the limit where the great majority of the agents work in the tier 1 , the agents in the tier 2 receive a share $\alpha$ of the total amount of wages.

Figure 3. In (a) the Lorentz curve for a given value of the relative wage (in black), and the gray curve shows all possible locus for the vertexes of the different Lorentz curves for different relative wages, all in the TVE case. In (b) us depicted the Gini index with respect number of agents in tier 2, for the TVE case (black line) and for the SNE case (gray line)

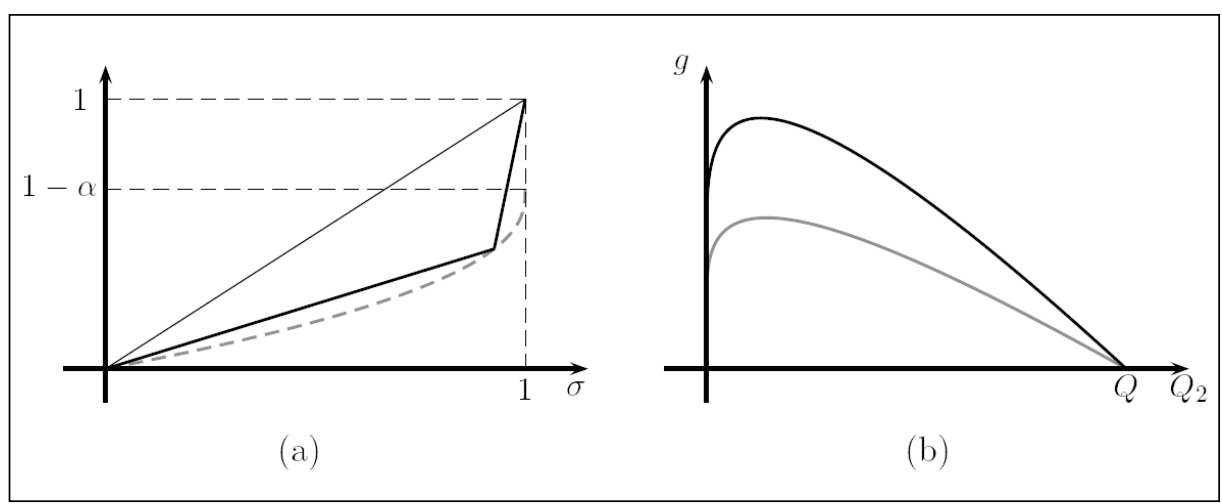

The Gini index is defined as the area between the Lorentz curve and the perfect equality curve divided by the total area under this second line. In our case, the Gini index takes the following form:

$$
g \equiv 2 \cdot \int_{0}^{1}(\sigma-g(\sigma)) \cdot d \sigma=\frac{d}{d+q}-\frac{d \cdot \frac{w_{b}}{w_{2}}}{d \cdot \frac{w_{b}}{w_{2}}+q}
$$


The difference among the two cases we have treated arise from the different dependences among $q_{1} / d$ and the relative wage $r_{w}$, given in (3.10) and (4.4). We can depict the Gini index with respect the fraction of workers of the tier 2, and the result s shown on figure 3 (b), for the TVE case (black line) and for the SNE case (gray line). We observe that, because the difference among wages is higher in the TVE case, also the Gini index is higher in this case.

In this picture we observe that Gini index for $Q \rightarrow 0$ is not 0 , meaning that in this limit the small number of agents in the tier 2 own an aggregated wage that is a proportion $\alpha$ of the total aggregated wage (this corresponds to $\sigma \rightarrow 1$ in the figure 3 (a)). We observe an initial increase of the Gini index when the number of workers of the tier 2 moves from 0. Surprisingly, more egalitarian is the distribution of the income (when $Q_{2}$ rises then $r_{w}$ rises and $w_{b}$ approaches to $w_{2}$ ), higher is the inequality index. The increase of agents in tier 2 generates more inequality than the convergence of the wages. Moreover, in this region, larger is the Gini index, the higher are both the utility of the rich and the poor people. This structure consists in few people with a very high income and a lot of people with very low wages that, instead of the incentive of the high difference of wages, tend to supply a low quantity of effort.

Interestingly, as the number of agents in the tier 2 increases there is a maximum in the index of Gini index. This point corresponds to the moment that the convergence of the wages compensates the inequality generated by the increase of the agents in the tier 2. Finally the Gini index decreases with the minimum wage, achieving 0 when all the agents belong to the second tier. In this point the overall real production is 0 , because there are no incentives to work, as we have seen before. Nevertheless, this region is Pareto inefficient, because as we have seen, increases in the number of agents in the tier 2 make the average utility of all the agents in our economy decreases.

\section{Conclusions}

The model we have presented helps us to understand the firm organization and the wage distribution among the different tiers of jobs within the same firm.

When variables that are important to determine the level of production are not observable by the firm owner he or she must establish a incentives system to ensure that the agents provide a certain level of effort. We have modelled this process as upgrading lotteries among the agents, where the probability of being upgraded depends stochastically on the effort supplied by the agents. This is a realistic assumption because in fact, the agents know that their success in the firm depends on the 
effort they supply. Agents in the same tier compete for the available vacancies. This competition makes them supply even though it is not directly observable.

The two cases we have analyzed produce very similar results. In fact, the capacity of the firm owner to choose a level effort or when this level effort is chosen by the agents produce few differences at the effort supplied and the production obtained by the firm owner. When there are only two values of effort available by the agents, because the election set is discrete, it is easy to make the agents supply effort. By the other hand, when the level effort is elected through a competition process among the agents, because the effort is continuous, the agents decide a local maximum of the function, lowering the effort supplied. The difference among the two cases depends essentially on the effort aversion, parameterized by $\beta$, that determines the importance the possibility of choosing a local maximum.

Competition among firms implies that the prices decrease until the moment that profits turn to be 0 . Because the 0 profits price is strongly related with the structure, the firms that produce similar goods will have similar structures and wages. Moreover, the technological changes in the productive process that change the organization towards the price minimizer scheme will be favored by the market, because the firms that initially adopt of this kind of technologies will have positive profits temporally.

We have seen that when the firms can be restructured, that is, if the productive process allows different organization schemes, the structure is changed in order to decrease the prices and then be more competitive. This process makes the firms more similar, because there is an unique organization scheme that minimizes the price. When it is applied at the whole economy level the election of the structure coincides with the maximization of the real wage of the tier 1 agents is maximized, and also their utility in the TVE case when $c=0$.

The sectors are better off when there are regulations that increase the expected quantity of high tier workers, for example by regulating the structure or the relative wage among tiers, because although they make 0 profits we have seen that the average utility is increased. Although this kind of regulations generate productive inefficiencies, they increase the nominal output and the welfare of the regulated sector, by rising the price of the good. Nevertheless, as we have seen, when these regulations applied to the whole economy the result is a decrease in the overall welfare, and the economy becomes inefficient.

Although the simplifying assumption of a firm (or the whole economy) organized only two tiers may seem too simplified, this model is a first step that has allowed us to obtain many interesting results. In fact, most of the models that consider more than one role usually consider only two roles. Models with workers and firms, with high- 
ly skilled and low skilled workers, models with producers and predators, models with landowners and peasants,..., are models that help us to explain some observed facts and that allow us to understand them better. Albeit the reality is far of being as simple as only having two roles, because there are a lot of different roles and people assuming many of them at the same time, the simple models allow us to analyze many effects observed in the reality.

Inequality indexes, like the Gini index, may not be a good indicator of the welfare of the people. As we have seen the Gini index is maximized (and then the inequality reaches its maximum) near the zone where the minimum real wage is also maximized. Moreover, the Gini index decreases as the proportion of tier 2 agents approximates to 1 , when the real wages of both the tier 1 and the tier 2 agents approach to 0 . Then, the imposition of certain equality laws sometimes imply low Gini indexes, but reduce the real wage and utility of all the agents in the economy, and then they leave the economy in a non Pareto efficient situation.

\section{REFERENCES}

[1] Akerlof, G. A. and J. Yellen (1990). «The Fair Wage-Effort Hypothesis and Unemployment», Quarterly Journal of Economics 105 (May); 255-283, 1990.

[2] Alchian. A. and H. Demsetz (1972). «Production, Information Costs, and Economic Organization», American Economic Review, 62, 777-95.

[3] Chandler, A. (1962). «Strategy and Structure: Chapters in the History of the Industrial Enterprise», MA: MIT press.

[4] Calvo, G. and S. Wellisz (1978). «Supervision, Loss of Control and the Optimal Size of the Firm», Journal of Political Economy, 86, 943-52. .

[5] Calvo, G. and S. Wellisz (1979). «Hierarchy, Ability, and Income Distribution», Journal of Political Economy, 87, 991-1010.

[6] Dickens, W. T.; L. F. Katz, K. Lang and L. H. Summers (1989). «A Employee Crime and the Monitoring Puzzle», Journal of Labor Economics 7 (July); 331-348, 1989.

[7] Green, J. R. and N. L. Stokey (1983). «A Comparison of Tournaments and Contracts», The Journal of Political Economy, Vol. 91, No. 3 (Jun., 1983), págs. 349-364.

[8] Holmstrom, B. (1982). «Moral Hazard in Teams», Bell Journal of Economics, 13, 324-40.

[9] Lazear, E. P. and S. Rosen (1981). «Rank-Order Tournaments as Optimum Labor Contracts», The Journal of Political Economy, Vol. 89, No. 5 (Oct., 1981), págs. 841-864.

[10] Nalebuff, B. J. and J. E. Stiglitz (1983). «Information, Competition, and Markets», The American Economic Review, Vol. 73, No. 2, Papers and Proceedings of the Ninety-Fifth Annual Meeting of the American Economic Association (May, 1983), págs. 278-283. 
[11] Qian, Y. (1994). «Incentives and Loss of Control in an Optimal Hierarchy», Review of Economic Studies. 61, 527-44.

[12] Ramsey, F. (1928). «A Mathematical Theory of Saving», Economic Journal, 38, December, 543-559.

[13] Ricardo, D. (1817). «On the Principles of Political Economy and Taxation», Cambridge: Cambridge University Press, 1951.

[14] Shapiro, C. and J. E. Stiglitz (1984). «Equilibrium Unemployment as a Worker Discipline Device», American Economic Review 84 (June): 433-444.

[15] Tirole, J. (1986). «Hierarchies and Bureaucracies: On the Role of Collusion in Organizations», Journal of Law, Economics and Organization, Oxford University Press, vol. 2(2), pages 181-214, Fall. 